have intentionally harmed oneself or attempted suicide, was described as highly risky. Doing so would diminish a person's social status in this context, exposing them to additional stressors during interactions within their community and with services. Consequently, people tended to hide mental distress and respond with behaviours less obviously linked to mental illness than self-harm.

Conclusion The stressors experienced by this deprived, ethnically diverse urban community acted to both make self-harm less common and reduce help-seeking following it, despite mental distress being common. When measuring mental health need in a population the influence of social context on reported outcomes needs to be considered to avoid reinforcing existing health inequalities.

\section{P65 HOW ARE OCCUPATIONAL HISTORIES ASSOCIATED WITH SELF-RATED HEALTH IN MIDDLE-AGED ADULTS? A CROSS-SECTIONAL ANALYSIS OF RETROSPECTIVE UK BIOBANK DATA}

${ }^{1} \mathrm{~A}$ Bhaskar*, ${ }^{1} \mathrm{~V}$ Katikireddi, ${ }^{2} \mathrm{C}$ Niedzwiedz, ${ }^{1} \mathrm{M}$ Mutambudzi, ${ }^{1} \mathrm{P}$ Flowers, ${ }^{3} \mathrm{~K}$ Hunt, ${ }^{1} E$ Demou. ${ }^{1}$ MRC/CSO SPHSU, University of Glasgow, Glasgow, UK; ${ }^{2}$ Mental Health and Wellbeing, University of Glasgow, Glasgow, UK; ${ }^{3}$ Institute for Social Marketing and Health, University of Stirling, Stirling, UK

\subsection{6/jech-2020-SSMabstracts. 158}

Background Employment and occupation can greatly influence one's life course, identity, resilience, health and health inequalities. At the same time, the working environment is rapidly changing, with flexible and agile working conditions and extended working lives, often resulting in 'non-traditional' career trajectories. We aimed to explore and identify common occupational history patterns and assess the associations between these patterns and self-rated health.

Methods We analysed baseline UK Biobank data to construct participants occupational histories using employment start/end dates to identify patterns of employment status during working life, with each year being categorised into different employment or employment gap states. We used sequence analysis, followed by optimal matching and cluster analysis methods to classify respondents' occupational history patterns. We produced Sequence Index Plots (SIPs) by gender and age groups. Theoretically derived occupational history patterns based on the SIPs were compared to the data-driven ones, to determine final patterns. Logistic regression models were run using occupational histories as explanatory variables for the health outcome of self-rated health (dichotomised: 'excellent/ good' versus 'fair/poor').

Results There was good agreement between the SIPs and cluster analysis; resulting in 3-5 different occupational history patterns per age/gender group, including continuous employment, employed then retired and employed with breaks for education and caring responsibilities as examples. Women aged 4049 had better overall self-rated health if continuously employed compared with those with a pattern that included employed/in education/caring responsibilities $\quad(\mathrm{OR}=0.80$; CI:0.69, 0.93), or those off work at some point due to ill health (OR: 0.68; CI: 0.59, 0.79). Men aged 40-49 reported worse self-rated health if employed and retired $(\mathrm{OR}=0.64$; CI:0.53, 0.78)) or were off work at some point due to ill health $(\mathrm{OR}=0.42 ; \mathrm{CI}: 0.31,0.57)$. Women aged $50-59$ and
60-69 tended to have better self-rated health if retired, even if their employment histories involved multiple gaps due to caring responsibilities or short-term employment. This was not the case for men; being continuously employed or continuously employed and then retired was generally associated with better self-rated health compared to groups with non-continuous employment.

Conclusion Continuous employment appeared to be associated with better self-rated health in men but not always for women. Our study is limited by the retrospective nature of the data and the limited representativeness of the study population. The modern working environment is rapidly changing, increasingly giving rise to 'non-traditional' career trajectories which might result in future adverse health impacts.

\section{P66 ESTIMATING INEQUALITIES IN MODERATE-VIGOROUS PHYSICAL ACTIVITY AMONG ADOLESCENTS IN ENGLAND AND THE US USING HURDLE MODELS}

S Scholes*, IS Mindell. Epidemiology and Public Health, University College London, London, UK

\subsection{6/jech-2020-SSMabstracts. 159}

Background Evidence is unclear on whether inequalities in average levels of moderate-vigorous physical activity (MVPA) reflect differences in participation, differences in the amount of time spent active, or both. Using self-reported data from $\mathrm{n}=4874$ adolescents aged $11-15$ in England (Health Survey for England: 2008, 2012, 2015) and $n=3065$ adolescents aged $12-15$ in the US (NHANES: 2007-16), we examined inequalities in these separate aspects for overall- and domainspecific MVPA.

Methods Socioeconomic position (SEP) was indexed by tertiles of equivalised household income (England) and the family income-to-poverty ratio (FIPR, US). Hurdle modelling is a novel way of analysing MVPA data with: (1) excessive zeros (non-participation), and (2) a continuous positively-skewed part (the amount of time active participants spend being active). We applied gender- and country-specific models to estimate inequalities in three aspects: (1) the probability of doing any MVPA, (2) the average hours-per-week (hpw) spent engaged in MVPA, and (3) the average hpw MVPA conditional on participation (MVPA-active). Using complete-case analyses adjusted for the complex survey design, absolute differences in MVPA (e.g. hpw) between adolescents in the highest versus lowest SEP were summarised using average marginal effects (AMEs) with 95\% Confidence Intervals (95\% CIs) after confounder adjustment (body mass index).

Results Inequalities in overall MVPA were observed in the US, but not in England. For example, the AMEs for girls in the US in the highest versus lowest SEP were $3.2 \mathrm{hpw}$ (95\% CI: 1.9 to $4.6 \mathrm{hpw}$ ) and $3.0 \mathrm{hpw}(1.7$ to $4.4 \mathrm{hpw})$ respectively for MVPA and MVPA-active. Inequalities in sports participation were evident for girls in both countries (AMEs for sports MVPA-active: England: $0.7 \mathrm{hpw}(0.1$ to $1.4 \mathrm{hpw})$; US: 2.5 hpw (1.4 to $3.6 \mathrm{hpw}$ ), and for boys in the US (AME: 2.0 hpw; 0.6 to $3.5 \mathrm{hpw}$ ). In contrast, boys in the highest versus lowest SEP spent less time on average in active travel (AME for MVPA: England: $-0.3 \mathrm{hpw}(-0.6$ to $0.1 \mathrm{hpw})$; US: -0.6 hpw $(-1.2$ to $0.1 \mathrm{hpw}))$; this finding mainly reflected the difference between SEP groups in the probability of doing any active travel. Girls in the US in the highest versus lowest SEP 
showed a similar propensity to do any active travel, but on average spent less time engaged in active travel. Findings were robust to different model specifications (e.g. using two-part models).

Conclusion Monitoring inequalities in PA requires assessing different aspects of the distribution within each domain.

\section{P67 USUAL PHYSICAL ACTIVITY AND SUBSEQUENT HOSPITAL USAGE OVER 20 YEARS IN A GENERAL POPULATION: THE EPIC-NORFOLK COHORT}

${ }^{1}$ RN Luben*, 'SA Hayat, ${ }^{2} \mathrm{NJ}$ Wareham, 'PD Pharoah, 'KT Khaw. 'Department of Public Health and Primary Care, Institute of Public Health, University of Cambridge, Cambridge, UK; ${ }^{2}$ MRC Epidemiology Unit, University of Cambridge School of Clinical Medicine, Cambridge, UK

\subsection{6/jech-2020-SSMabstracts. 160}

Background UK government spending on health has risen on average by $3.7 \%$ per year since 1948, outpacing economic growth over the period, with approximately a half of this expenditure used for hospitals. While physical activity interventions have been reported to reduce hospital stays, it is not clear if usual physical activity patterns may be associated with subsequent hospital use independently of other lifestyle factors. We examined the relationship between reported usual physical activity, change in usual physical activity and subsequent admissions to hospital and time spent in hospital for 11228 men and 13786 women aged 40-79 years in the general population over two sequential 10 -year follow-up period taking into account demographic and lifestyle factors.

Methods Participants from EPIC-Norfolk, a British prospective population-based cohort study were followed for 20 years (1999-2019) using record linkage to document hospital usage. Total physical activity was estimated by combining workplace and leisure time activity reported in a baseline lifestyle questionnaire and repeated with independent measures in a subset at a second time point approximately 12 years later.

Results Compared to those reporting no physical activity, participants who were the most active had a lower likelihood of spending more than 20 days in hospital odds ratio (OR) 0.75 (95\% confidence interval (CI) 0.67-0.83) over the next 10 years after multivariable adjustment for age, sex, smoking status, education, social class and body mass index. Similar results were seen for 10-year follow-up after the second time point OR 0.60 (95\% CI 0.50-0.72). Participants reporting any activity had a mean of 0.42 fewer hospital days per year between 1999 and 2009 compared to inactive participants, an estimated potential saving to the National Health Service (NHS) of $£ 247$ per person per year, or approximately $7 \%$ of UK health expenditure. Participants who remained physically active or became active 12 years later had lower risk of subsequent hospital usage than those who remained inactive or became inactive, $\mathrm{p}$ trend $<0.001$.

Conclusion Usual physical activity in this middle-aged and older population predicts lower future hospitalisations - time spent in hospital and number of admissions independently of behavioural and sociodemographic factors. Small feasible differences in usual physical activity in the general population may potentially have a substantial impact on hospital usage and costs.

\section{P68 ABSTRACT WITHDRAWN}

P69 FAMILY-BASED PHYSICAL ACTIVITY PROMOTION:
RESULTS FROM THE FAMILIES REPORTING EVERY STEP
TO HEALTH (FRESH) PILOT RANDOMISED CONTROLLED
TRIAL

${ }^{1}$ EMF van Sluijs ${ }^{*},{ }^{1} \mathrm{HE}$ Brown, ${ }^{2} \mathrm{E}$ Coombes, ${ }^{3} \mathrm{C}$ Hughes, ${ }^{2} \mathrm{AP}$ Jones, ${ }^{1} \mathrm{KL}$ Morton, ${ }^{1} \mathrm{JM}$ Guagliano. ${ }^{1}$ UKCRC Centre for Diet and Activity Research (CEDAR) and MRC Epidemiology Unit, University of Cambridge, Cambridge, UK; ${ }^{2}$ Norwich Medical School, University of East Anglia, Norwich, UK; ${ }^{3}$ Centre for Family Research, University of Cambridge, Cambridge, UK

\subsection{6/jech-2020-SSMabstracts. 161}

Background Family physical activity (PA) promotion holds promise, but there is little high quality research evaluating its potential and impact. Following successful feasibility assessment and adaption, this pilot study assessed the acceptability of FRESH, a child-led family-based PA intervention delivered online, and explored preliminary effectiveness.

Methods In a three-armed pilot randomised controlled trial (prospectively registered: ISRCTN12789422), 41 families (with 7-11-year-old index child) were allocated to a standard care control (CON), 'pedometer' (PED), or 'family' (FAM) group of the Self-Determination Theory-guided FRESH intervention. All family members in PED and FAM received pedometers and generic walking information; FAM additionally received access to the FRESH website, enabling participants to select step challenges, log steps, and track progress as they virtually globetrotted. All family members were eligible to participate; follow-up occurred 8-weeks and 52-weeks post-baseline. During home visits, research assistants assessed physical (e.g., fitness), psychosocial (e.g., social support), and behavioural (e.g., device-measured family PA) measures. Process evaluation questionnaires assessed acceptability of the intervention and accompanying evaluation. Semi-structured focus groups were conducted and website engagement explored.

Results Of 41 families recruited (149 participants; 4.0 1.0 (mean \pm SD) people/family), $40 \quad(98 \%)$ and 36 (88\%) were retained at 8-week and 52-weeks follow-up, respectively. Compared to CON and PED, a greater percentage of FAM children self-reported doing more family PA (CON: 35\%, PED: 45\%, FAM: 83\%) and found FRESH fun (CON: 93\%, PED: 81\%, FAM: 94\%). Higher mean $( \pm S D)$ scores were reported by parents in FAM for improved PA awareness (3.6 \pm 0.6 vs. $3.2 \pm 0.7)$ and increased family PA $(3.0 \pm 0.8$ vs. $2.5 \pm 0.8)$ compared to PED. Approximately $82 \%$ of FAM children wanted to keep using the FRESH website and 93\% found it easy to use. Focus groups revealed FAM families enjoyed choosing weekly step challenges and were capable of identifying ways of meeting daily steps goals. In children, there were no notable between-group differences found for change in minutes in moderate-to-vigorous PA (MVPA) at 8 or 52 weeks. In contrast, change in MVPA minutes differed between adults in the FAM group and those in PED or CON groups (FAM vs CON: 9.4 [95\%CI: 0.4, 18.4]; FAM vs PED: 15.3 [95\%CI: 6.0, 24.5]; PED vs CON: -5.8 [95\%CI: -15.1, 3.3]), however this was not maintained at 52 -weeks.

Conclusion Preliminary process evaluation findings related to the FRESH intervention and evaluation were promising, 